\title{
Classification of Imbalance Data using Tomek Link (T-Link) Combined with Random Under-sampling (RUS) as a Data Reduction Method
}

Elhassan $\mathrm{AT}^{1}$, Aljourf $\mathbf{M}^{1}$, Al-Mohanna $\mathrm{F}^{2,3}$, Shoukri $\mathbf{M}^{2,3^{*}}$

${ }^{1}$ Oncology Center, King Faisal Specialist Hospital and Research Center, Saudi Arabia

${ }^{2}$ Department of Cell Biology, Research Center, King Faisal Specialist Hospital and Research Center, Saudi Arabia

${ }^{3}$ College of Medicine, Al-Faisal University, Saudi Arabia

\begin{abstract}
The problem of classifying subjects into disease categories is of common occurrence in medical research. Machine learning tools such as Artificial Neural Network (ANN), Support Vector Machine (SVM) and Logistic Regression (LR) and Fisher's Linear Discriminant Analysis (LDA) are widely used in the areas of prediction and classification. The main objective of these competing classification strategies is to predict a dichotomous outcome (e.g. disease/healthy) based on several features.

Like any of the well-known statistical inferential models; machine learning tools are faced with a problem known as "class imbalance". A data set is imbalanced if the classification categories are not approximately equally represented. When learning from highly imbalanced data, most classifiers are affected by the majority class leading to an increase in the false negative rate. Increased interests in applying machine learning techniques to "real-world" problems, whose data are characterized by severe imbalance, have emerged as can be seen in numerous publications in medicine and biology.

Predictive accuracy, a popular choice for evaluating performance of a classifier, might not be appropriate when the data is imbalanced and/or when the costs of different errors vary markedly.

In this paper, we use the T-Link algorithm in the preprocessing phase as a method of data cleaning in order to remove noise. We combine T-Link with other sampling method such as RUS, ROS and Synthetic Minority Technique (SMOTE) in order to maintain a balanced class distribution. Classification was then utilized using several ML algorithms such as ANN, RF and LR. Classifiers performance was evaluated using several performance measures deemed more appropriate for classifying data with sever imbalance. These methods are applied to arterial blood pressures data and Ecoli2 data set.
\end{abstract}

Using TLink in combination with RUS and SMOTE demonstrated a superior performance compared to resampling techniques such among different classification algorithms such as SVM, ANN, RF and LR.

Keywords: Logistic regression; Neural network; Support vector machine; Imbalance data; T-Link

\section{Introduction}

Most of data in the real-word are imbalance in nature. This situation occurs when the distribution of the target class is not uniform among the different class levels. Classification of this type of data is one of the most challenging problems in the field of machine learning and has recently gained a great deal of interest [1]. This is because most of the known machines learning algorithms were developed with an optimal goal of maximizing the overall accuracy, which is the percentage of correct predictions made by a classifier. This results in classifiers with a high accuracy but very low sensitivity towards the positive class [2]. Therefore, the optimal goal needs to be shifted toward maximizing the sensitivity of positive class and negative class separately rather than focusing on the overall accuracy. Several methods were developed to overcome this problem; these methods include methods based on sampling techniques, cost-sensitive learning, Ensemble learning, Feature selection and algorithmic modification [3].

Classification has many applications in the medical field. Classifying patients in to different medical diagnosis using different machine learning algorithms has attracted the interest of many researchers in clinical medicine. This is because most of the diagnosis techniques can be transformed in to intelligent data classification systems where diagnosis can be made based on some features such as medical finding.

With increased applications of medical informatics algorithms and software, medical databases can now be utilized to extract different features associated with different types of diagnoses. Therefore, automated diagnosis systems can be developed to aid clinicians and biomedical scientists throughout the diagnosis process. In terms of machine learning, building an automated diagnosis classification system is an optimization problem with an ultimate objective of increasing the diagnostic accuracy. However, this problem becomes difficult when the target class has imbalance distribution of the class levels.

Splitting the whole dataset into training and testing datasets using random sampling technique without any adjustment of the class distribution will not solve the imbalance problem. This is because the training data will have the same distribution of the original data set,

*Corresponding author: Shoukri M, Department of Cell Biology, Research Center King Faisal Specialist Hospital and Research Center, P.O. Box 3345 11211-Riyadh, Saudi Arabia, Tel: +966509491454; E-mail: shoukri@kfshrc.edu.sa

Received March 31, 2017; Accepted May 30, 2017; Published June06, 2017

Citation: Elhassan AT, Aljourf M, Al-Mohanna F, Shoukri M (2017) Classification of Imbalance Data using Tomek Link (T-Link) Combined with Random Undersampling (RUS) as a Data Reduction Method. Global J Technol Optim S1: 111. doi: 10.4172/2229-8711.S1:111

Copyright: (C) 2017 Redjaimia A. This is an open-access article distributed under the terms of the Creative Commons Attribution License, which permits unrestricted use, distribution, and reproduction in any medium, provided the original author and source are credited. 
a problem that will persist during the learning phase. There are two methods to deal with this situation; one is sampling-based method and the other is a cost-sensitive method.

Sampling methods tend to adjust the prior distribution of the minority and majority classes in the training data to have a balanced class distribution. Re-sampling techniques can be classified into basic sampling techniques and advanced sampling techniques. Basic sampling techniques include methods such as Random under-sampling (RUS) of majority class, Random over-sampling (ROS) of minority class, and a hybrid of both. On the other hand, advanced sampling techniques are basically based on the idea of a guided sampling approach which has been utilized using special methods. These methods include Tomek Link (T-Link) [4], Synthetic Minority Oversampling Technique (SMOTE) [5], One-Sided Selection (OSS) [6-27], Neighborhood Cleaning Rule (NCR) [7], Edited Nearest Neighbor Rule (ENN) [8], Condensed Nearest Neighbor (CNN) [9] and Bootstrap-based Over Sampling (Boot-OS) [10] etc.

Cost-sensitive learning is used in situations where misclassification cost varies with the type of errors. In this case we are faced with two issues: Skewed class distribution and different misclassification cost. This type of learning is used in domains such as fraud detection [11], medical diagnosis and risk management [12-14]. Cost-sensitive learning can be classified into direct cost-sensitive learning and Meta-Learning Methods (MLM). The MLM are used when direct implementation of misclassification cost into the learning algorithm is not provided. Implementation of these methods is utilized through meta-learning thresholding and meta-learning sampling [15-18].

Ensemble learning is a machine learning method that uses multiple learners-called base learners-to learn from multiple bootstrap samples generated from the training data set. It has a strong generalizability as compared to machine learners that use a single learner because of its ability to boost weak learners in to stronger learners and finally aggregate the results and make the predictions based on the majority of votes. Ensemble learning method is based on the work done by Breiman [19]. It includes methods such as bagging and boosting.

Feature selection is a method of feature reduction where only relevant features are used in the learning process. It is usually used with high-dimensional data [20]. However, in case of unbalanced data, the process of feature selection need to be adjusted to select features that lead to more class separability [21]

Algorithmic modification approach works on the algorithmic level where the learning algorithm is adjusted to fit the imbalance data context. This method can be implemented by three methods; proposing new splitting criteria [22], adjusting the distribution reference in the Decision Tree (DT), and adjusting the Offset Entropy [23].

In this paper we focused on sampling techniques using basic and advanced methods. We hypothized that applying a guided data reduction method such as T-Link followed by a random under-sampling (RUS) will improve the classification model sensitivity toward the minority class compared to other sampling methods.

Different sampling techniques were utilized to emphasize the comparative performance of different classification algorithms such as Artificial Neural Networks (ANN), Support Vector Machine (SVM), Random Forest (RF) and Logistic Regression (LR).

Classification accuracy was evaluated using different measures such as weighted accuracy, sensitivity, specificity, G-mean, F-statistics, $\mathrm{NPV}$, and area under the ROC curve.
The paper is structured as follows: In Section 2 we introduce a brief description of sampling-based methods used in this study. Section 3 introduces the research methodology and framework. Section 4 presents two experimental studies where the different studying sampling techniques were applied to different ML algorithms. $\mathrm{R}$ studio and Weka 3.8 for machine learning were utilized.

\section{Sampling-based Methods}

\section{Basic sampling methods}

Over sampling: It is a non-heuristic method that balances the class distribution by duplicating examples of minority class. There are two types of over-sampling; a Random Over-Sampling (ROS) in which examples to be duplicated are randomly chosen; Focus Over-Sampling (FOS) where duplication process includes only examples that are located in the borders between the two classes.

Under sampling: It is a non-heuristic method where subset of majority class is chosen to create a balanced class distribution. There are two types of under-sampling; Radom Under-Sampling (RUS) where some of the majority class examples are removed randomly; and Focused Under-Sampling (FUS) that excludes majority examples that are located on the borders between the two classes [24]

The advantage of this method is that; the elimination of some examples could significantly reduce the size of the data and therefore decrease the run-time cost especially in case of big data. However, ignoring some examples from the majority class might lead to loss of some important information that might contribute to the learning process [25].

Other methods such as Tomek-Link [26], Condensed Nearest Neighbor and One-sided selection were proposed to improve the performance of random undersampling.

\section{Advanced sampling methods}

Tomek Link (T-Link): T-Link is a method of under-sampling developed by Tomek [26]. It is considered as an enhancement of Nearest-Neighbor Rule (NNR). The algorithm work as follows:

Let $\mathrm{x}$ be an instance of class $\mathrm{A}$ and $\mathrm{y}$ an instance of class $\mathrm{B}$.

Let $\mathrm{d}(\mathrm{x}, \mathrm{y})$ be the distance between $\mathrm{x}$ and $\mathrm{y}$.

$(\mathrm{x}, \mathrm{y})$ is a T-Link, if for any instance $\mathrm{z}, \mathrm{d}(\mathrm{x}, \mathrm{y})<\mathrm{d}(\mathrm{x}, \mathrm{z})$ or $\mathrm{d}(\mathrm{x}, \mathrm{y})$ $<\mathrm{d}(\mathrm{y}, \mathrm{z})$

If any two examples are T-Link then one of these examples is a noise or otherwise both examples are located on the boundary of the classes.

T-Link method can be used as a method of guided under-sampling where the observations from the majority class are removed.

Several researches use T-link as a method of under sampling [28-31].

Synthetic Minority Oversampling Technique (SMOTE): SMOTE is an advance method of over-sampling developed by Chawala [4]. It aims to enrich the minority class boundaries by creating artificial examples in the minority class rather replicating the existing examples to avoid the problem of overfitting. The algorithm works as follows:Let $\mathrm{A}$ be the minority class and let $\mathrm{B}$ be the majority class.

Then, for each observation $\mathrm{x}$ belongs to class A, a k-nearest neighbors of $\mathrm{x}$ are identified, 
A few neighbors are randomly selected (the number of neighbors depends on the rate of over-sampling),

Artificial observations are then generated and spread along the line joining the $\mathrm{x}$ to its nearest neighbors.

Several methods have been developed to improve the original SMOTE algorithm such as dealing with nominal features. New methods include SMOTE-NC (Synthetic Minority Over-sampling Technique Nominal Continuous) and SMOTE-N (Synthetic Minority Over-sampling Technique Nominal). These methods can be considered as a generalization of the original SMOTE algorithm to handle data sets with mixed Features (continuous and nominal).

Several works have been done in this field. Estabrooks et al. [32] proposed a multiple re-sampling method that selects the most appropriate re-sampling rate. Jo et al. [33] introduced a cluster-based over-sampling method which considers the between-class imbalance and within-class imbalance simultaneously. Guo et al. [34] focused on the hard examples of the majority and minority classes using the boosting algorithm, and then generated new synthetic examples from hard examples and add them to the data sets. Han et al. [35] presented two new minority over-sampling methods based on SMOTE method, borderline-SMOTE1 and borderline-SMOTE2, where only the minority examples near the borderline are oversampled. These approaches achieve better sensitivity rate and F-value as compared to SMOTE and random over-sampling methods.

\section{Methodology}

This study can be divided into three phases, Figures 1 and 2:

\section{The pre-processing phase}

This phase is dived into sub phases as follows:

Data reduction: In this phase T-Link method was utilized as methods of data reduction. Applying this method noise was removed from the majority class in order to improve the classification process. Therefore, this method is considered as a guided under-sampling where only noise data are removed from the majority class.

\section{Resampling}

Different resampling techniques were applied to the reduced data as well as the original (imbalanced) data sets. Resampling methods include, RUS, ROS, SMOTE. The output of this phase is a group of six resampled data sets which are: RUS, ROS, SMOTE, TLink/RUS, TLink/ROS and TLink/SMOTE.

\section{Normalization}

Numeric variables were normalized using the following formula:

$$
X_{\text {new }}=\frac{X-X_{\min }}{X \max -X \min }
$$

Therefore, all values will be in the range $[0,1]$.

Imputation of missing data: Missing data was treated using the most frequent value for nominal variables and the median for numeric variables.

\section{Feature selection}

Features section was utilized using the CfsSubsetEval algorithm. This algorithm evaluates the worth of a subset of attributes by considering the individual predictive ability of each feature along with the degree of redundancy between them. The subsets of features which are the highly correlated with the target class while having low intercorrelation were selected.

\section{The classification phase}

Different classification algorithms were utilized to predict the target class given a set of features. Algorithms include Support Vector Machine (SVM), Artificial Neural Networks (ANN), Random Forest (RF) and Logistic Regression (LR). Following is a brief description of each algorithm.

\section{Support Vector Machine (SVM)}

The support vector machine (SVM) is a widely used technique for data classification and regression. It has become an important topic in machine learning and pattern recognition. Not only it has a better theoretical foundation, practical comparisons have also shown that it is superior to the ANN.

The SVM technique was first developed by Vapnik and his group in former AT\&T Bell Laboratories. The original idea is to use a linear separating hyper-plane which maximizes the distance between two

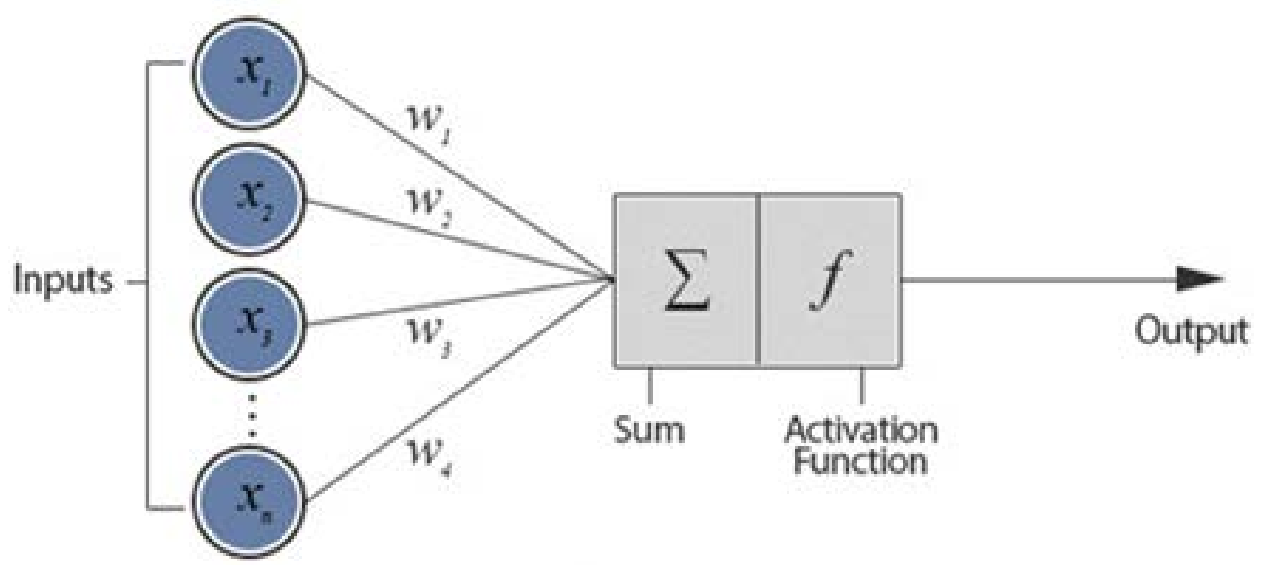

Figure 1: Simple illustration of ANN. 


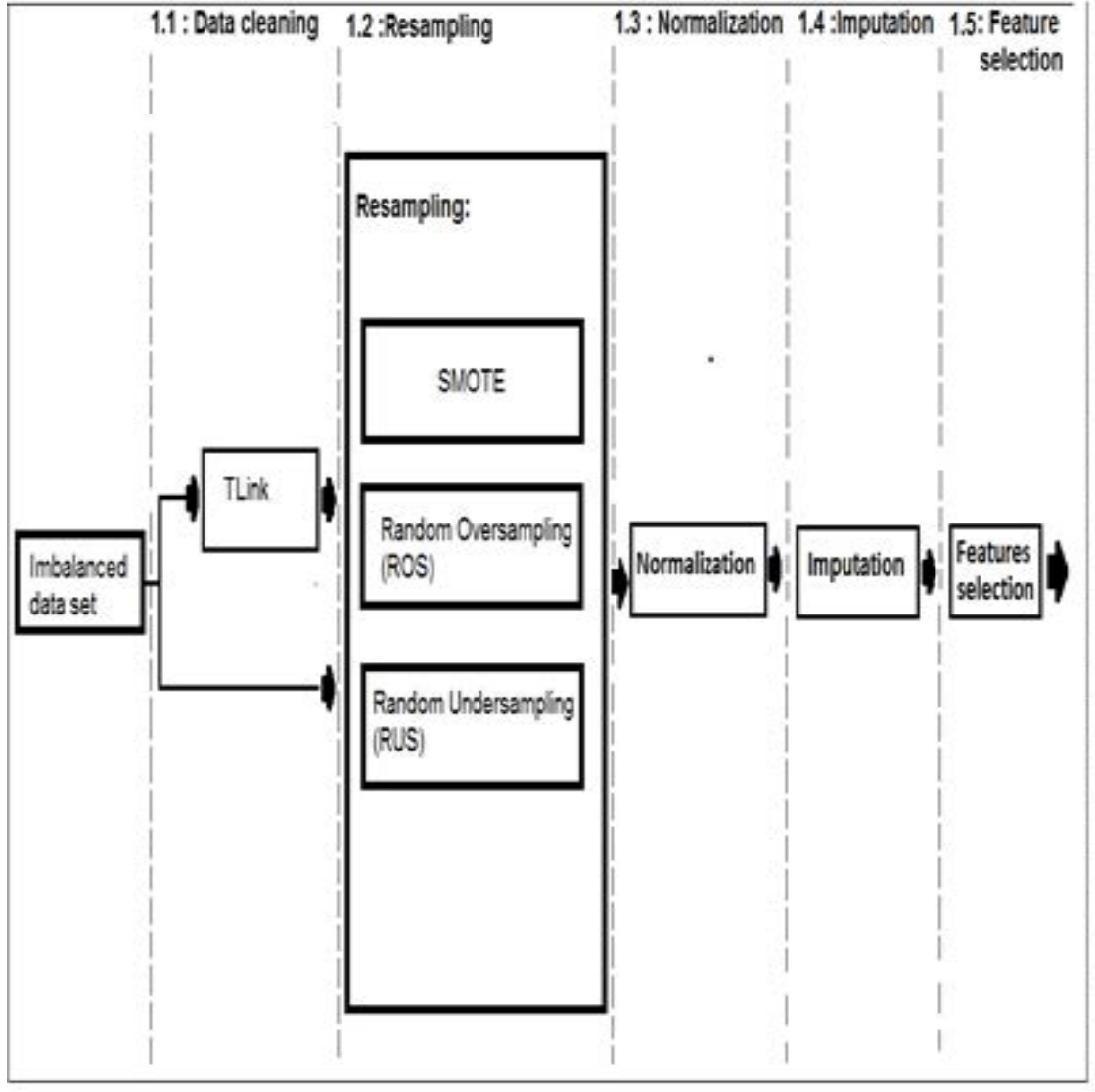

Phase 1: Preprocessing

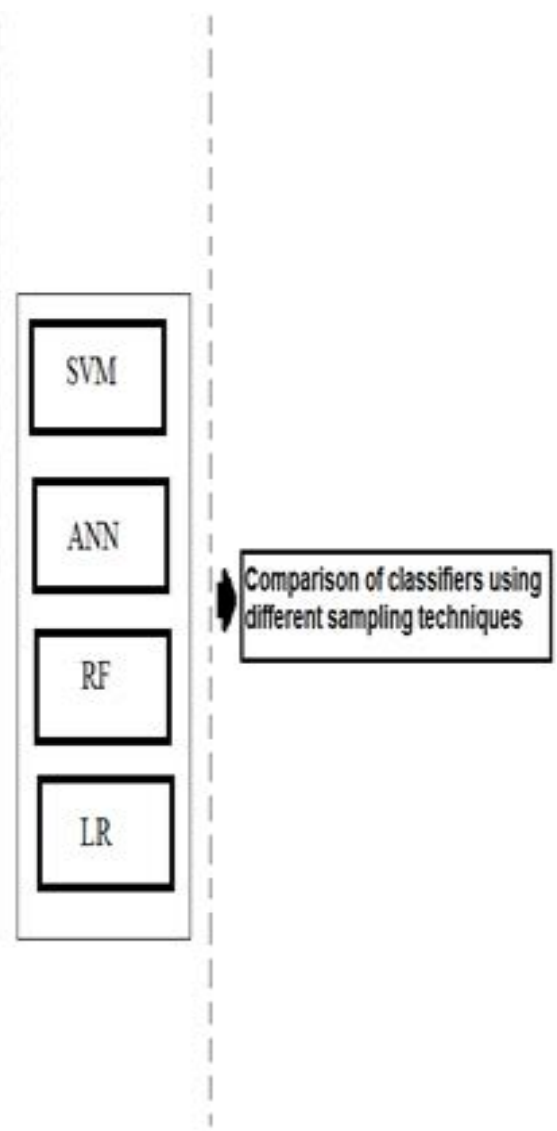

Phase 2:Classification Phase 3: Comparison

Figure 2: Research methodology.

classes to create a classifier. Therefore the SVM is also known as "hyper-plane classifier".

Briefly, SVM (SVM) [36-39] is machine-learning derived classifiers which map a vector of predictors into a higher dimensional plane through either linear or non-linear kernel functions. In binary classification problem, the two groups say $\{-1\}$ and $\{+1\}$ are separated in a higher-dimension hyperplane accordingly to structural risk minimization principle. The objective is to find a linear separating hyper-plane:

$$
\mathrm{w}^{\prime} \varnothing(\mathrm{x})+\mathrm{b}=0
$$

Constructed from a vector $\mathrm{x}$ of predictors mapped into a higher dimension feature space by a nonlinear feature function $\varnothing$, a vector $\mathrm{w}$ of weights and bias offset $b$, that classifies all the observation yi in one of the two groups $\{-1 ;+1\}$.

\section{Artificial Neural Networks (ANN)}

ANNs provide an analytical alternative to conventional techniques which are often limited by strict assumptions of normality, linearity, variable independence as required by the LR. Because an ANN can capture many kinds of relationships it allows the user to quickly and relatively easily model phenomena which otherwise may have been very difficult or impossible to explain.

Let $\mathrm{x}$ denote the vector of $\mathrm{n}$ predictors, and $\mathrm{w}$ is the vector of input weights. The function $f($.$) is a processing activation function for the$ output [40]. Activation functions are one of the general linear, logistic, exponential or Gaussian function families. Several topologies of Neural Networks (NN) can be used in binary classification problems. Two of the most used NN are the Multilayer Perceptron (MLP) and the Radial Basis Function (RBF). The main differences between these two $\mathrm{NN}$ reside in the activation functions of the hidden layer: For the MLP the activation function belongs, generally, to a linear

Neural Networks (NN) methods have been used extensively in classification problems.

$$
f_{j}(x)=\sum_{i=1}^{n} w_{i j} x_{i}
$$

Or logistic activation function family:

$$
f(x)=\frac{1}{1+\exp (-x)}
$$

For the RBF function the activation function belongs to the Gaussian family:

$$
f_{j}(x)=\exp \left[-\frac{1}{2}\left(x-\mu_{j}\right)^{\prime} \sum_{j}^{-1}\left(x-\mu_{j}\right)\right]
$$

A NN is generally trained in a set of iterations (epochs) for a subset 
(epochs) for a subset of the data (train set) and tested for the remained subset (test set). The vector of synaptic weights (w) of the $\mathrm{NN}$ is upgraded in each iteration in way to maximize the correct classification errors; either a function of the sum of squares of the errors for a continuous criterion: $S S E=\frac{1}{2} \sum_{i=1}^{n}\left(\gamma_{i}-\hat{\gamma}_{i}\right)^{2}$ or the Cross-entropy error function for a binary criterion:

\section{Random Forest (RF)}

It takes a bootstrap sample from the data and fits a classification or regression tree. When building decision trees, each time a split is considered, a random sample of $\mathrm{m}$ predictors is chosen as split candidates from the full set of $\mathrm{p}$ predictors.

The split is allowed to use only one of those m predictors. A fresh sample of $\mathrm{m}$ predictors is taken at each split, and typically we take $\mathrm{m}=$ $\sqrt{ } p$. Where, $\mathrm{p}$ is number of predictors.

The best split on the selected $\mathrm{m}$ variables is then found and tree is grown. The final predication is made by voting for classification and by averaging for regression [19].

\section{Logistic Regression (LR)}

Binomial Logistic regression (LR) models the probability of occurrence of one (success) of the two classes of dichotomous criterion. A linear combination of predictors is used to fit a Logit transformation of the probability of success for each subject $(\pi i)$ as

$$
\operatorname{Ln}\left[\hat{\pi}_{i} /\left(1-\hat{\pi}_{i}\right)\right]=w_{0}+w_{1} X_{1 i}+\ldots . \beta w_{n} X_{n i}
$$

Regression coefficients are fitted by maximum likelihood estimation, and by solving the Logit in order to $\pi \mathrm{i}$ the probability of success for each subject is estimated as

$$
\hat{\pi}_{i}=\frac{e^{w_{0}+w_{1} X_{1 i}+\ldots w_{n} X_{n i}}}{1+e^{w_{0}+w_{1} X_{1 i}+\ldots w_{n} X_{n i}}}
$$

If for subject $i$ the estimated probability is greater than 0.5 (or other user pre-defined threshold value), the subject is classified into the success group; otherwise, it is classified into the failure group. Detailed descriptions can be found in [41].

The comparison phase: In this phase, the improvement of model sensitivity towards the minority class using different sampling techniques was assessed. Model performance was evaluated using different measures such as weighted accuracy, sensitivity, specificity, precision and AUC. Following is a brief definition of each measure.

$$
\begin{aligned}
& \text { Sensitivity: The True Positive rate }(\mathrm{TP})=\frac{T P}{T P+F N} \\
& \text { Specificity: The True Negative rate }(\mathrm{TN})=\frac{T N}{F P+T N}=1-\text { fprate } \\
& \text { Precision: Positive predictive value }(\mathrm{PPV})=\frac{T P}{T P+F P} \\
& \text { Negative Predictive Value (NPV) }=\frac{T N}{F N+T N} \\
& F-\text { statistics }=\frac{2 * \text { precision } * \text { sensitivity }}{\text { precision }+ \text { sensitivity }} \\
& G-\text { mean }=\sqrt{\text { sensitivity } * \text { specificity }} \\
& \text { Weighted accuracy }=0.5 * \text { Sensitivity }+0.5 * \text { Specificity }
\end{aligned}
$$

AUC: A single scalar value represents the excepted performance of ROC curve. AUC calculates the area under the ROC curve. It calculates the probability that the classifier will rank a randomly chosen positive instance higher than a randomly chosen negative instance.

$$
A U C=\frac{\text { sensitivity }+ \text { specificity }}{2}
$$

AUC has an advantage over other performance measures; that is; AUC is invariant to relative class distributions, and class-specific error costs.

\section{Experimental Studies}

For any classifier, there is always a tradeoff between true positive rate and true negative rate; and the same applies for recall and precision. In the case of learning extremely imbalanced data, usually the rare class is of great interest. In many applications such as drug discovery and disease diagnosis, it is desirable to have a classifier that gives high prediction accuracy over the minority (positive) class (Acc + ), while maintaining reasonable accuracy for the majority (negative) class (Acc-). In this section, we applied the previously discussed techniques to improve the classifier predictive ability of the positive class using two imbalanced data sets. Following is a description for the two studying data sets.

\section{Data sets}

Familial data: This is a highly structured clustered familial data that has two levels of hierarchy, where blood measurements were taken on parents (level two) and their offspring (level one) together with other anthropometric features [42]. Familial data sets are notorious of having considerable "within-cluster" correlation due to the homogeneous nature of family members. The goal is to classify the offspring blood pressure status based on parents BP and other anthropometric features.

The data set contains 223 families with a mean number of siblings equal to 3 siblings per family.

The outcome variable in this data set is a binary variable which is offspring blood pressure status (High (+) vs. Normal (-)) (Table 1). The data has 12 attributes described as follows:

1. Father age

2. Mother age

3. Father systolic blood pressure

\begin{tabular}{|c|c|}
\hline Variables & Median (range=(25th, 75th))/n (\%) \\
\hline Father age & $58(47,65)$ \\
\hline Mother age & $53(44,61)$ \\
\hline Father systolic blood pressure & $140(120,155)$ \\
\hline Father diastolic blood pressure & $80(70,90)$ \\
\hline Mother systolic blood pressure & $145(130,170)$ \\
\hline Mother diastolic blood pressure & $85(75,95)$ \\
\hline Mother arm girth & $10(9.8,11.8)$ \\
\hline Father arm girth & $9.8(9,10.5)$ \\
\hline sibling age & $23(14,33)$ \\
\hline sibling sex & $550(88 \%)$ \\
\hline Male & $76(12 \%)$ \\
\hline Female & $9.2(8,10.3)$ \\
\hline sibling arm girth & $4(3,5)$ \\
\hline Number of siblings & \\
\hline
\end{tabular}

Table 1: Patient's characteristics. 
4. Father diastolic blood pressure

5. Mother systolic blood pressure

6. Mother diastolic blood pressure

7. Mother arm girth

8. Father arm girth

9. sibling age

10. sibling sex

11. sibling arm girth

12. Number of siblings

Ecoli2 data: This data is about protein localization sites. This data is created and maintained by Kenta Nakai [43].

The outcome variable is a binary class that represents the protein localization site. It has two levels \{positive and negative\}. The positive examples belong to class pp and the negative examples belong to the rest. This data contains 363 instances and 7 attributes (Table 2). Following is a description for the attributes of this data set:

mcg: McGeoch's method for signal sequence recognition.

gvh: von Heijne's method for signal sequence recognition.

lip: von Heijne's Signal Peptidase II consensus sequence score. Binary attribute.

\begin{tabular}{|c|c|}
\hline Attribute & Domain \\
\hline Mcg & {$[0.0,0.89]$} \\
\hline Gvh & {$[0.16,1.0]$} \\
\hline Lip & {$[0.48,1.0]$} \\
\hline Chg & {$[0.5,1.0]$} \\
\hline Aac & {$[0.0,0.88]$} \\
\hline Alm1 & {$[0.03,1.0]$} \\
\hline Mcg & {$[0.0,0.89]$} \\
\hline Gvh & {$[0.16,1.0]$} \\
\hline Lip & {$[0.48,1.0]$} \\
\hline Chg & {$[0.5,1.0]$} \\
\hline Aac & {$[0.0,0.88]$} \\
\hline Alm1 & {$[0.03,1.0]$} \\
\hline Class $\{$ positive, negative & Class $\{$ positive, negative \\
\hline
\end{tabular}

Table 2: Patient's characteristics. chg: Presence of charge on N-terminus of predicted lipoproteins. Binary attribute.

aac: Score of discriminant analysis of the amino acid content of outer membrane and periplasmic proteins.

alm1: Score of the ALOM membrane spanning region prediction program.

alm2: Score of ALOM program after excluding putative cleavable signal regions from the sequence.

\section{Results}

\section{Familial data}

Applying T-Link data reduction methods, only 34 (9\%) cases was removed from the minority class.

Results showed an improved performance when applying T-Link as a data cleaning method prior to the different sampling techniques. Combining T-Link with under-sampling has showed the best performance among all the studying classification algorithms. However, although the specificity (prediction accuracy of majority class) has been slightly decreased; models showed a reasonable increase in sensitivity (accuracy of the minority class), G-mean and F-statistics (Tables 3a-3d).

Following is the performance improvement using T-Link/undersampling as compared to the original imbalanced data using different classification algorithms:

SVM: Performance measures such as F-statistic, G-mean, sensitivity and weighted accuracy has been increased from $7 \%, 19 \%$, $4 \%$ and $52 \%$ to $45 \%, 74 \%, 74 \%$ and $74 \%$ in the original data as compared to T-Link/under-sampled data respectively. AUC has also been increased from $69 \%$ to $78 \%$ in the original data and T-Link/undersampled data respectively.

ANN: Using original imbalanced data, F-statistic, G-mean, sensitivity and weighted accuracy were $30 \%, 45 \%, 26 \%$ and $59 \%$. However, using T-Link/under-sampling performance measures have been increased to $41 \%, 72 \%, 78 \%$ and $72 \%$ respectively. AUC was also improved from $70 \%$ to $79 \%$ using original data and T-Link/undersampled data respectively.

Random Forest: using T-Link/under-sampling, F-statistic, G-mean, sensitivity and weighted accuracy have been increased from $25 \%$, $38 \%, 15 \%$ and $57 \%$ using original data to $37 \%, 67 \%, 67 \%$ and $67 \%$. AUC has also been improved from $71 \%$ to $75 \%$ using the original data and T-Link/under-sampling respectively.

\begin{tabular}{|c|c|c|c|c|c|c|c|c|}
\hline Support Vector Machine & Original & T-link & SMOTE & SMOTE/T-Link & Over-sampling & Over/ T-link & Under-sampling & Under/ T-link \\
\hline Sensitivity & 0.037 & 0.037 & 0.481 & 0.481 & 0.519 & 0.556 & 0.667 & 0.741 \\
\hline Specificity & 1 & 0.976 & 0.752 & 0.714 & 0.708 & 0.696 & 0.602 & 0.74 \\
\hline Accuracy & 0.862 & 0.844 & 0.713 & 0.681 & 0.681 & 0.676 & 0.612 & 0.74 \\
\hline Weighted accuracy & 0.519 & 0.506 & 0.617 & 0.598 & 0.613 & 0.626 & 0.635 & 0.74 \\
\hline Precision & 1 & 0.2 & 0.245 & 0.22 & 0.23 & 0.234 & 0.22 & 0.323 \\
\hline NPV & 0.861 & 0.861 & 0.896 & 0.891 & 0.898 & 0.903 & 0.915 & 0.944 \\
\hline F-statistics & 0.071 & 0.063 & 0.325 & 0.302 & 0.318 & 0.33 & 0.33 & 0.449 \\
\hline G-mean & 0.192 & 0.19 & 0.602 & 0.586 & 0.606 & 0.622 & 0.634 & 0.74 \\
\hline AUC & 0.695 & 0.69 & 0.705 & 0.72 & 0.736 & 0.754 & 0.745 & 0.784 \\
\hline
\end{tabular}

Table 3a: Performance measures of different sampling techniques using different leaning algorithms (familial data set) (Support Vector Machine (SVM) 


\begin{tabular}{|c|c|c|c|c|c|c|c|c|}
\hline Artificial Neural Network & Original & T-link & SMOTE & SMOTE/ T-Link & Over-sampling & Over/ T-link & Under-sampling & Under/ T-link \\
\hline Sensitivity & 0.259 & 0.333 & 0.444 & 0.481 & 0.556 & 0.481 & 0.704 & 0.778 \\
\hline Specificity & 0.919 & 0.894 & 0.807 & 0.857 & 0.72 & 0.807 & 0.689 & 0.658 \\
\hline Accuracy & 0.824 & 0.814 & 0.755 & 0.803 & 0.697 & 0.761 & 0.691 & 0.676 \\
\hline W-accuracy & 0.589 & 0.614 & 0.626 & 0.669 & 0.638 & 0.644 & 0.697 & 0.718 \\
\hline Precision & 0.35 & 0.346 & 0.279 & 0.361 & 0.25 & 0.295 & 0.275 & 0.276 \\
\hline NPV & 0.881 & 0.889 & 0.897 & 0.908 & 0.906 & 0.903 & 0.933 & 0.946 \\
\hline F-statistics & 0.298 & 0.34 & 0.343 & 0.413 & 0.345 & 0.366 & 0.396 & 0.408 \\
\hline G-mean & 0.488 & 0.546 & 0.599 & 0.642 & 0.633 & 0.624 & 0.697 & 0.716 \\
\hline AUC & 0.7 & 0.74 & 0.726 & 0.76 & 0.715 & 0.724 & 0.71 & 0.79 \\
\hline
\end{tabular}

Table 3b: Performance measures of different sampling techniques using different leaning algorithms (familial data set)-Artificial Neural Network (ANN)

\begin{tabular}{|c|c|c|c|c|c|c|c|c|}
\hline Logistic regression & Original & T-link & SMOTE & SMOTE/ T-Link & Over-sampling & Over/ T-link & Under-sampling & Under/ T-link \\
\hline Sensitivity & 0.037 & 0.037 & 0.407 & 0.407 & 0.37 & 0.407 & 0.407 & 0.667 \\
\hline Specificity & 1 & 1 & 0.82 & 0.783 & 0.853 & 0.795 & 0.776 & 0.752 \\
\hline Accuracy & 0.862 & 0.862 & 0.761 & 0.729 & 0.781 & 0.739 & 0.723 & 0.739 \\
\hline W-accuracy & 0.519 & 0.519 & 0.614 & 0.595 & 0.611 & 0.601 & 0.592 & 0.709 \\
\hline Precision & 1 & 1 & 0.275 & 0.239 & 0.303 & 0.25 & 0.234 & 0.31 \\
\hline NPV & 0.861 & 0.861 & 0.892 & 0.887 & 0.887 & 0.889 & 0.887 & 0.931 \\
\hline F-statistics & 0.071 & 0.071 & 0.328 & 0.301 & 0.333 & 0.31 & 0.297 & 0.424 \\
\hline G-mean & 0.192 & 0.192 & 0.578 & 0.565 & 0.562 & 0.569 & 0.562 & 0.708 \\
\hline AUC & 0.74 & 0.73 & 0.712 & 0.722 & 0.737 & 0.73 & 0.704 & 0.792 \\
\hline
\end{tabular}

Table 3c: Performance measures of different sampling techniques using different leaning algorithms (familial data set)- Logistic Regression (LR).

\begin{tabular}{|c|c|c|c|c|c|c|c|c|}
\hline Random Forest & Original & T-link & SMOTE & SMOTE/ T-Link & Over-sampling & Over/ T-link & Under-sampling & Under/ T-link \\
\hline Sensitivity & 0.148 & 0.222 & 0.259 & 0.37 & 0.296 & 0.296 & 0.63 & 0.667 \\
\hline Specificity & 0.994 & 0.988 & 0.901 & 0.851 & 0.95 & 0.957 & 0.708 & 0.671 \\
\hline Accuracy & 0.872 & 0.878 & 0.809 & 0.782 & 0.856 & 0.862 & 0.697 & 0.67 \\
\hline W-accuracy & 0.571 & 0.605 & 0.58 & 0.611 & 0.623 & 0.626 & 0.669 & 0.669 \\
\hline Precision & 0.8 & 0.75 & 0.304 & 0.294 & 0.5 & 0.533 & 0.266 & 0.254 \\
\hline PVP & 0.874 & 0.883 & 0.879 & 0.89 & 0.89 & 0.89 & 0.919 & 0.923 \\
\hline F-statistics & 0.25 & 0.343 & 0.28 & 0.328 & 0.372 & 0.381 & 0.374 & 0.367 \\
\hline G-mean & 0.384 & 0.468 & 0.483 & 0.561 & 0.531 & 0.532 & 0.668 & 0.669 \\
\hline AUC & 0.71 & 0.723 & 0.706 & 0.702 & 0.713 & 0.721 & 0.749 & 0.754 \\
\hline
\end{tabular}

Table 3d: Performance measures of different sampling techniques using different leaning algorithms (familial data set)- Random Forest (RF).

Logistic regression: LR also showed an improved performance using T-Link/under-sampling as compared to original data. F-statistic, G-mean, sensitivity and weighted accuracy have been increased from $7 \%$ to $42 \%, 19 \%$ to $71 \%$, and $4 \%$ to $66 \%$ and from $52 \%$ to $71 \%$ using the original data and T-Link/under-sampled data respectively. AUC has also showed an improved performance using T-Link/under-sampling (74\% vs. $79 \%)$.

Tables 3a-3d and Figure 3 shows comparative performance results of different classifiers using different sampling techniques.

\section{Ecoli2 data}

Applying T-Link data reduction methods, only 36 (13\%) cases was removed from the minority class. Results showed a comparable performance when applying T-Link prior to the different sampling techniques. Combining T-Link with under-sampling has also showed a comparable performance as compared to other sampling methods. Applying resampling methods, models showed a reasonable increase in sensitivity, G-mean and F-statistics, (Tables $4 \mathrm{a}-4 \mathrm{~d}$ ). Following is the performance improvement using T-Link/undersampling as compared to the original imbalanced data using different classification algorithms:

SVM: The best results were shown using Tlink/undersampling as a re-sampling method. Performance measures such as F-statistic, G-mean, sensitivity and weighted accuracy has been increased from $63 \%, 74 \%, 58 \%$ and $76 \%$ to $923 \%, 923 \%, 92 \%$ and $92 \%$ in the original data as compared to T-Link/under-sampled data respectively. AUC has also been increased from $91 \%$ to $95 \%$ in the original data and T-Link/ under-sampled data respectively.

ANN: The best results were shown using Tlink/SMOTE resampling method. Using original imbalanced data, F-statistic, G-mean, sensitivity and weighted accuracy were $83 \%, 90 \%, 85 \%$ and $90 \%$. However, using T-Link/SMOTE performance measures have been increased to $96 \%, 96 \%, 94 \%$ and $96 \%$ respectively. AUC was also improved from $95 \%$ to $97 \%$ using original data and T-Link/undersampled data respectively.

Random Forest: The best results were shown using T-link/SMOTE 
Citation: Elhassan AT, Aljourf M, Al-Mohanna F, Shoukri M (2017) Classification of Imbalance Data using Tomek Link (T-Link) Combined with Random Under-sampling (RUS) as a Data Reduction Method. Global J Technol Optim S1: 111. doi: 10.4172/2229-8711.S1:111

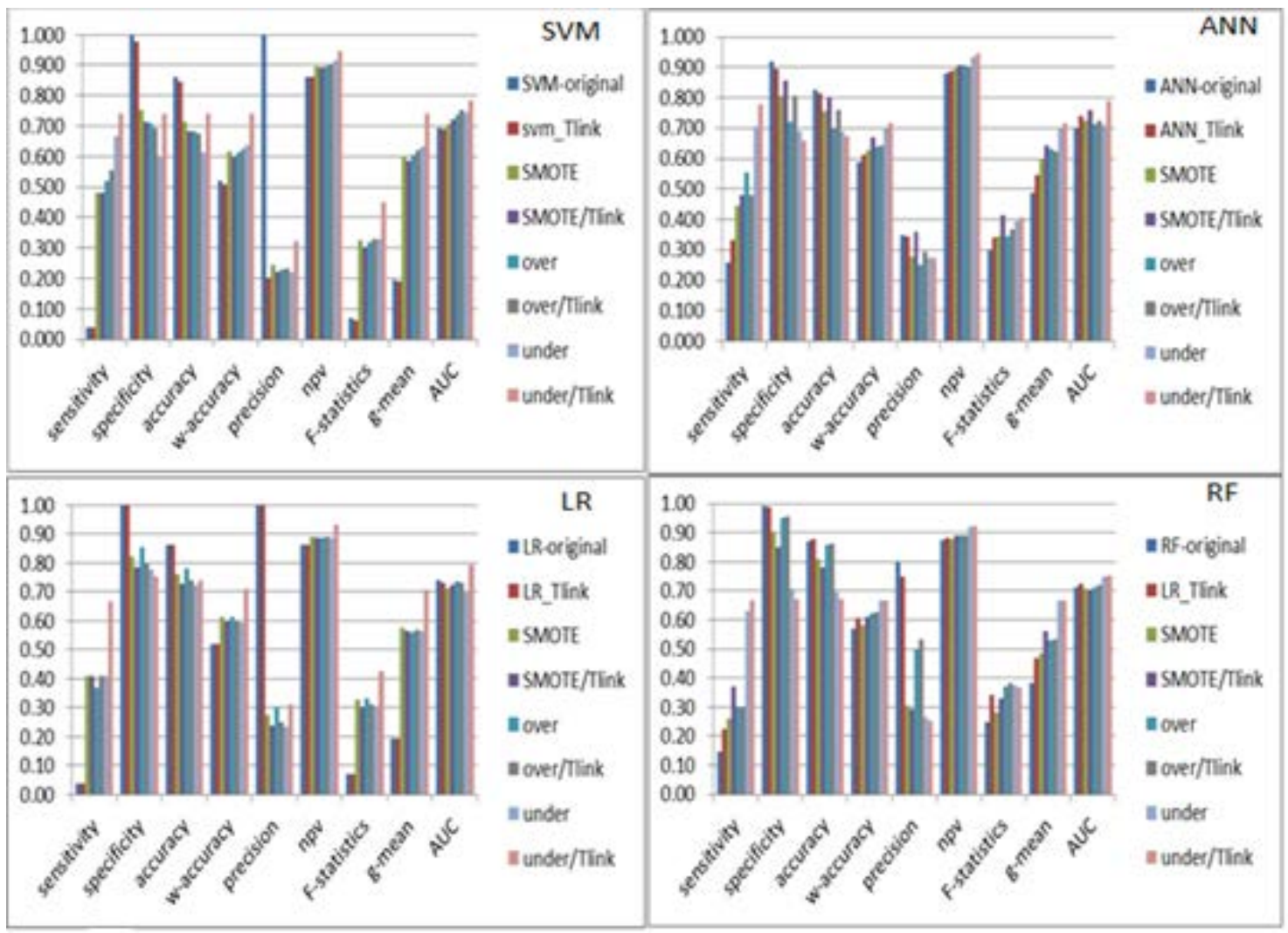

Figure 3: Performance of different ML algorithms using different sampling techniques (family data).

\begin{tabular}{|c|c|c|c|c|c|c|c|c|}
\hline Support Vector Machine & Original & T-Link & SMOTE & SMOTE/T-Link & Over-sampling & Over/T-Link & Under-sampling & under/T-Link \\
\hline Sensitivity & 0.577 & 0.673 & 0.929 & 0.91 & 0.931 & 0.883 & 0.942 & 0.923 \\
\hline Specificity & 0.951 & 0.964 & 0.918 & 0.899 & 0.895 & 0.88 & 0.885 & 0.923 \\
\hline Accuracy & 0.893 & 0.918 & 0.923 & 0.904 & 0.913 & 0.882 & 0.913 & 0.923 \\
\hline W-accuracy & 0.764 & 0.818 & 0.924 & 0.905 & 0.913 & 0.882 & 0.913 & 0.923 \\
\hline Precision & 0.682 & 0.778 & 0.895 & 0.871 & 0.899 & 0.883 & 0.891 & 0.923 \\
\hline NPV & 0.925 & 0.94 & 0.946 & 0.93 & 0.929 & 0.88 & 0.939 & 0.923 \\
\hline F-statistics & 0.625 & 0.722 & 0.912 & 0.89 & 0.915 & 0.883 & 0.916 & 0.923 \\
\hline G-mean & 0.741 & 0.805 & 0.924 & 0.905 & 0.913 & 0.882 & 0.913 & 0.923 \\
\hline AUC & 0.913 & 0.922 & 0.945 & 0.953 & 0.932 & 0.934 & 0.96 & 0.95 \\
\hline
\end{tabular}

Table 4a: Performance measures of different sampling techniques using different leaning algorithms (Ecoli2 data set)-Support Vector Machine (SVM).

\begin{tabular}{|c|c|c|c|c|c|c|c|c|}
\hline Artificial Neural Network & Original & TLink & SMOTE & SMOTE/TLink & Overs-samplig & Over/TLink & Under-sampling & under/TLink \\
\hline Sensitivity & 0.846 & 0.808 & 0.929 & 0.942 & 0.964 & 0.924 & 0.942 & 0.923 \\
\hline Specificity & 0.965 & 0.917 & 0.938 & 0.981 & 0.942 & 0.949 & 0.962 & 0.942 \\
\hline Accuracy & 0.946 & 0.899 & 0.934 & 0.964 & 0.953 & 0.937 & 0.952 & 0.933 \\
\hline W-accuracy & 0.905 & 0.862 & 0.933 & 0.962 & 0.953 & 0.937 & 0.952 & 0.933 \\
\hline Precision & 0.815 & 0.646 & 0.918 & 0.974 & 0.943 & 0.948 & 0.961 & 0.941 \\
\hline NPV & 0.972 & 0.962 & 0.947 & 0.958 & 0.963 & 0.926 & 0.943 & 0.925 \\
\hline F-statistics & 0.83 & 0.718 & 0.924 & 0.958 & 0.953 & 0.936 & 0.951 & 0.932 \\
\hline G-mean & 0.904 & 0.86 & 0.933 & 0.961 & 0.953 & 0.937 & 0.952 & 0.933 \\
\hline AUC & 0.95 & 0.92 & 0.95 & 0.97 & 0.96 & 0.96 & 0.96 & 0.95 \\
\hline
\end{tabular}

Table 4b: Performance measures of different sampling techniques using different leaning algorithms (Ecoli2 data set)-Artificial Neural Network (ANN). 
Citation: Elhassan AT, Aljourf M, Al-Mohanna F, Shoukri M (2017) Classification of Imbalance Data using Tomek Link (T-Link) Combined with Random Under-sampling (RUS) as a Data Reduction Method. Global J Technol Optim S1: 111. doi: 10.4172/2229-8711.S1:111

Page 9 of 11

\begin{tabular}{|c|c|c|c|c|c|c|c|c|}
\hline Logistic Regression & Original & TLink & SMOTE & SMOTE/TLink & Over-samplig & Oversampling/TLink & Under-sampling & Under-/TLink \\
\hline Sensitivity & 0.615 & 0.615 & 0.923 & 0.91 & 0.924 & 0.906 & 0.92 & 0.923 \\
\hline Specificity & 0.965 & 0.96 & 0.899 & 0.899 & 0.891 & 0.88 & 0.884 & 0.923 \\
\hline Accuracy & 0.911 & 0.905 & 0.909 & 0.904 & 0.908 & 0.893 & 0.903 & 0.923 \\
\hline W-accuracy & 0.79 & 0.788 & 0.911 & 0.905 & 0.908 & 0.893 & 0.903 & 0.923 \\
\hline Precision & 0.762 & 0.744 & 0.873 & 0.871 & 0.895 & 0.883 & 0.888 & 0.923 \\
\hline NPV & 0.932 & 0.93 & 0.94 & 0.93 & 0.921 & 0.903 & 0.92 & 0.923 \\
\hline F-statistics & 0.681 & 0.674 & 0.897 & 0.89 & 0.909 & 0.894 & 0.905 & 0.923 \\
\hline G-mean & 0.771 & 0.769 & 0.911 & 0.905 & 0.907 & 0.893 & 0.903 & 0.923 \\
\hline AUC & 0.934 & 0.92 & 0.949 & 0.95 & 0.935 & 0.936 & 0.954 & 0.95 \\
\hline
\end{tabular}

Table 4c: Performance measures of different sampling techniques using different leaning algorithms (Ecoli2 data set)-Logistic Regression (LR).

\begin{tabular}{|c|c|c|c|c|c|c|c|c|}
\hline Random Forest & Original & TLink & SMOTE & SMOTE/TLink & Over-samplig & Over/TLink & Under-sampling & Under-/TLink \\
\hline Sensitivity & 0.808 & 0.827 & 0.91 & 0.923 & 0.906 & 0.899 & 0.904 & 0.923 \\
\hline Specificity & 0.982 & 0.993 & 0.962 & 0.99 & 0.913 & 0.888 & 0.962 & 0.942 \\
\hline Accuracy & 0.955 & 0.966 & 0.94 & 0.962 & 0.91 & 0.893 & 0.933 & 0.933 \\
\hline W-accuracy & 0.895 & 0.91 & 0.936 & 0.957 & 0.91 & 0.893 & 0.933 & 0.933 \\
\hline Precision & 0.894 & 0.956 & 0.947 & 0.986 & 0.913 & 0.889 & 0.959 & 0.941 \\
\hline NPV & 0.965 & 0.968 & 0.935 & 0.945 & 0.906 & 0.897 & 0.909 & 0.925 \\
\hline F-statistics & 0.848 & 0.887 & 0.928 & 0.954 & 0.909 & 0.894 & 0.931 & 0.932 \\
\hline G-mean & 0.891 & 0.906 & 0.936 & 0.956 & 0.91 & 0.893 & 0.932 & 0.933 \\
\hline AUC & 0.96 & 0.97 & 0.98 & 0.99 & 0.97 & 0.972 & 0.96 & 0.983 \\
\hline
\end{tabular}

Table 4d: Performance measures of different sampling techniques using different leaning algorithms (Ecoli2 data set)-Random Forest (RF).

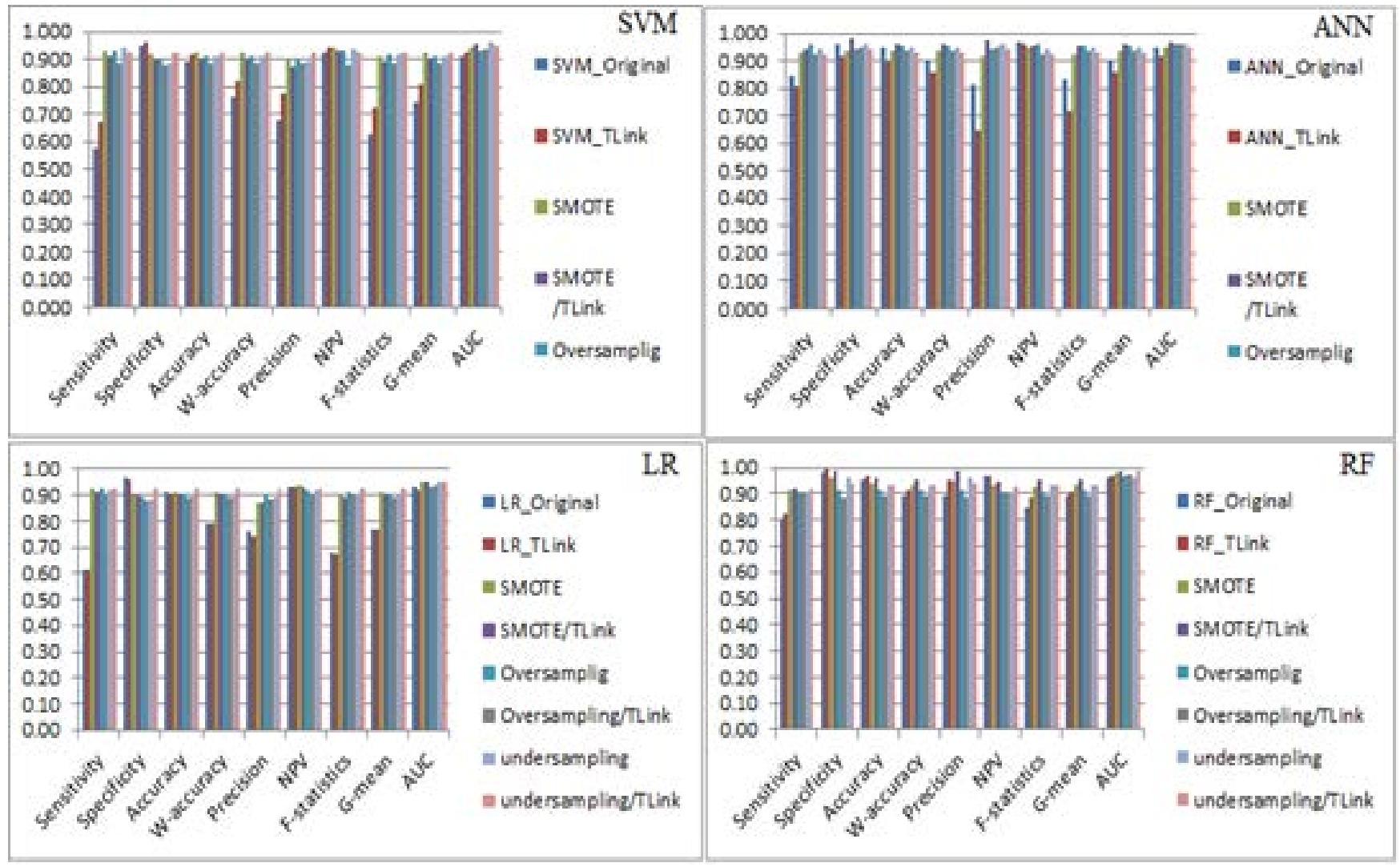

Figure 4: Performance of different ML algorithms using different sampling techniques (Ecoli2 data set). 
re-sampling method. Using T-Link/under-sampling, F-statistic, G-mean, sensitivity and weighted accuracy have been increased from $85 \%, 89 \%, 81 \%$ and $89 \%$ using original data to $95 \%, 96 \%, 92 \%$ and $96 \%$. AUC has also been improved from $96 \%$ to $99 \%$ using the original data and T-Link/under-sampling respectively.

Logistic regression: The best results were shown using T-link/ undersampling re-sampling method. LR also showed an improved performance using T-Link/under-sampling as compared to original data. F-statistic, G-mean, sensitivity and weighted accuracy have been increased from $68 \%$ to $92 \%, 77 \%$ to $92 \%$, and $61 \%$ to $92 \%$ and from $79 \%$ to $92 \%$ using the original data and T-Link/under-sampled data respectively. AUC has also showed an improved performance using T-Link/under-sampling (93\% vs.95\%). Tables $4 \mathrm{a}-4 \mathrm{~d}$ and Figure 4 shows comparative performance results of different classifiers using different sampling techniques.

\section{Discussion}

Imbalance data distribution has a significant impact on the performance of standard classification algorithms that assumes equivalent class distribution. Applying the standard classification algorithms; without any adjustment; results in a classification bias towards the majority class. Several methods have been proposed to adjust the standard classification process in the presence of imbalance class problem. Some of these methods work in the algorithmic-level while other methods work in the data-level [44].

Many experiments have been done using different adjustment methods. However, conclusions were contractive with no absolute winner. Some researchers showed superiority of sampling techniques while others recommend cost-sensitive and ensemble methods as optimal solutions. For examples, Anand et al. and Li et al. recommended sampling techniques as an optimal solution $[45,46]$; while McCarthy et al. and Liu et al. showed a superiority of cost-sensitive method[47,48]. On the other hand, Quinlan and Thomas showed superiority of ensemble methods while CIeslak and Marcelline showed best performance using algorithmic techniques.

Given all advantages of sampling techniques such as simplicity and transportability etc.; they are still presented with some limitations such as information loss, class overlapping, over fitting and prediction bias towards minority class. It is also difficult to distinguish between the minority class and noise observation.

However, Wing et al. proposed a solution for the problem of information loss using the diversified sensitivity-based undersampling. In this method samples of the majority class are clustered to capture the distribution information and hence improve the diversity of the resampling. Finally a balanced set of samples which achieves the highest sensitivity is selected.

Advance sampling techniques such as T-Link have partially solved the problem by removing noise. However, the problem of imbalance class distribution is still present.

In this paper, we recommend the use of T-Link as a method of data cleaning followed by random under-sampling as an option for data cleaning.

Removing noise observations from majority class followed by random sampling reduces the chance of information loss and therefor improves the performance of the classification algorithm.

Results of this investigation showed a superior performance using T-Link prior to the resampling process.
Familial data set showed the superiority of undersampling after T-link using all ML algorithms. Ecoli2 data set showed a comparable performance using all resampling methods. However, among all resampling methods, T-Link/undersampling the best performance using SVM and LR while SMOTE/T-Link showed the best performance using RF and ANN.

Removing noise observations from majority class followed by random sampling reduces the chance of information loss and therefor improves the performance of the classification algorithm.

Our finding comes along with the finding by Hartayuni and Santi where they showed that using combine sampling method; in their case; (SMOTE and T-Link) has an improved performance in terms of F-statistic, G-mean and AUC using SVM as a classification algorithm[46]. By looking at (Table 4a), our data showed that using SMOTE and T-Link as a combined sampling method has a better performance over T-Link sampling and the original data. However, in our study combing T-Link with under-sampling showed the best results.

Cost-sensitive techniques have a robust sense but still limited to some learning algorithms such as decision trees and neural networks. Cost-sensitive learning can lead to a classification biased toward positive class because the positive class is assigned high misclassification error cost. Consequently, the decision region created by the algorithm will be located far away from the positive instance. In this case, the learning algorithm needs to be modified in order to push the decision boundaries toward the positive samples to avoid classification bias. Another problem using cost-sensitive learning is that the real cost is usually unknown.

Ensemble methods are more casual and need minimal setting. They run through much iteration that makes them more accurate. The problem of this method is that they need large data and they are limited to decision trees.

Algorithmic methods are effective but they are limited by development complexity and have limited options.

\section{Conclusion}

Learning from imbalanced data sets is an important topic, both practically and for research.

When learning from imbalanced data, the classifiers are usually overwhelmed by the majority class, so the minority class examples tend to be misclassified.

The data presented in this study was severely imbalanced. Our main objective was to demonstrate that an improvement in the classification performance of the standard classification algorithms towards prediction of positive (rare) class using preprocessing techniques could be achieved.

Using T-Link method as a tool of data cleaning to remove noise prior to resampling methods such as random undersampling and SMOTE identified the best performer among different classification algorithms such as SVM, ANN, RF and LR. For this highly structured data set, under-sampling techniques showed superiority in performance compared to over-sampling techniques among all studied classifiers.

\section{Conflict of Interest}

The authors declare no conflict of interest.

\section{Acknowledgement}

The authors acknowledge the constructive comments made by two anonymous reviewers. 
Citation: Elhassan AT, Aljourf M, Al-Mohanna F, Shoukri M (2017) Classification of Imbalance Data using Tomek Link (T-Link) Combined with Random Under-sampling (RUS) as a Data Reduction Method. Global J Technol Optim S1: 111. doi: 10.4172/2229-8711.S1:111

\section{References}

1. Qiang Yang, Xindong Wu (2006) 10 Challenging Problems in Data Mining Research. International Journal of Information Technology \& Decision Making 4: 597-604.

2. Gu J, Zhou Y, Zuo X (2007) Making Class Bias Useful: A Strategy of Learning from Imbalanced Data. Lecture Notes in Computer Science, Intelligent Data Engineering and Automated Learning - IDEAL.

3. Choi MJ (2010) A Selective Sampling Method for Imbalanced Data Learning on Support Vector Machines, Graduate Theses, loawa State University.

4. Tomek Ivan (1976) An Experiment with the Edited Nearest-Neighbor Rule IEEE Transactions on Systems. Man, and Cybernetics 6: 448-452.

5. Chawla NV, Bowyer KW, Hall LO, Kegelmeyer WP (2002) SMOTE: Synthetic Minority Over-sampling Technique. Journal of Artificial Intelligence Research 16: $321-357$.

6. Kubat M, Matwin S (1997) Addressing the curse of imbalanced training sets: One-sided selection. ICML, pp: 179-186.

7. Laurikkala J (2001) Improving Identification of Difficult Small Classes by Balancing Class Distribution. AIME, LNAI 2101, pp: 63-66.

8. Wilson DL (1972) Asymptotic Properties of Nearest Neighbor Rules Using Edited Data. IEEE Transactions on Systems. Man, and Communications 2: 408-421.

9. Angiulli F, Bucci $P(2005)$ Fast condensed nearest neighbor rule Appearing in Proceedings of the 22nd International Conference on Machine Learning, Bonn, Germany.

10. Jingbo Z, Eduard H (2007) Active Learning for Word Sense Disambiguation with Methods for Addressing the Class Imbalance Problem, Proc. of the Joint Conference on Empirical Methods in Natural Language Processing and Computational Natural Language Learning, pp: 783-790.

11. Shen A, Tong R, Deng Y (2007) Application of Classification Models on Credit Card Fraud Detection. International Conference on Service Systems and Service Management, IEEE.

12. Domingos $P$ (1999) Metacost: A general method for making classifiers costsensitive. In Proceedings of the 5th international conference on knowledge discovery and data mining, pp: 155-164.

13. Pazzani M, Merz C, Murphy P, Ali K, Hurne K, et al. (1994) Reducing misclassification costs: Knowledge-intensive approaches to learning from noisy data. In Proceedings of the Eleventh International Conference on Machine Learningm, pp: 217-225.

14. Zadrozny B, Langford J, Abe N (2003) Cost-sensitive learning by costproportionate example weighting. In Proceedings of the $3 \mathrm{rd}$ IEEE international conference on data mining (ICDM'03), pp: 435-442.

15. Sheng VS, Ling CX (2006) Thresholding for Making Classifiers Cost-sensitive. In Proceedings of the 21st National Conference on Artificial Intelligence pp: 476-481.

16. Chai X, Deng L, Yang Q, Ling CX (2004) Test-Cost Sensitive Naïve Bayesian Classification. In Proceedings of the Fourth IEEE International Conference on Data Mining, UK

17. Thai-Nghe Nguyen, Gantner Zeno, Schmidt-Thieme Lars (2010) Cost-Sensitive Learning Methods for Imbalanced Data. Inf. Syst. \& Machine Learning Lab, Univ. Hildesheim, Germany.

18. Breiman L (1996) Bagging Predictors, Machine Learning, Kluwer Academic Publishers 24: 123-140.

19. Guyon I, Elissee A (2003) An introduction to variable and feature selection. Journal of Machine Learning Research 3: 1157-1182.

20. Chawla N V, Japkowicz N, (2004) Editorial: Special Issue on Learning from Imbalanced Data Sets. SIGKDD Explorations 6: 1.

21. Dietterich T, Kearns M, Mansour Y, (1996) Applying the weak learning framework to understand and improve C4.5. In Proc 13th International Conference on Machine Learning, pp: 96-100.

22. Marcellin S, Zighed D A, Ritschard G (2006) Detection of breast cancer using an asymmetric entropy measure. In: COMPSTAT-Proced. In Computational Statistics, pp: 975-982
23. Drummond C, Holte RC (2003) C4.5, class imbalance, and cost sensitivity: Why under sampling beats over-sampling. In Workshop on Learning from Imbalanced Datasets II, held in conjunction with ICML.

24. Japkowicz N (2000) Learning from imbalanced data sets: a comparison of various strategies. AAAI Tech Report WS-00-05.

25. Tomek Ivan (1976) An Experiment with the Edited Nearest-Neighbor Rule IEEE Transactions on Systems, Man, and Cybernetics 6: 448-452.

26. Thai-Nghe N, Do TN, Schmidt-Thieme L (2000) Learning Optimal Threshold on Resampling Data to Deal with Class Imbalance. Proc. of the 8th IEEE International Conference on Computing

27. Batista G, Carvalho A, Monard MC (2000) Applying One-sided Selection to Unbalanced Datasets. In Proceedings of MICAI, 315-325.

28. Andrew Estabrooks, Taeho Jo, Nathalie Japkowicz (2004) Multiple Resampling Method for Learning from Imbalanced Data Sets. Computational Intelligence 20: 18-36.

29. Taeho Jo, Nathalie Japkowicz (2004) Class Imbalances versus Small Disjunction. Sigkdd Explorations 6: 40-49.

30. Hongyu Guo, Herna L Viktor (2004) Learning from Imbalanced Data Sets with Boosting and Data Generation: The Data Boost-IM Approach $\square$. Sigkdd Explorations 6: 30-39.

31. Hui Han, Wen-Yuan Wang, Bing-Huan Mao (2005) Borderline-SMOTE: A New Over-Sampling Method in Imbalanced Data Sets Learning. pp: 878-887.

32. Vapnik, Vladimir N (1995) The Nature of Statistical Learning Theory

33. Noble W (2006) What is a support vector machine?. Nature Biotechnology 24 1565-1567.

34. Cortes C, Vapnik V (1995) Support-vector network. Machine Learning 20 273-297.

35. Cristianini N, Taylor S (2000) An Introduction to Support Vector Machines. Cambridge University Press, Cambridge, UK.

36. Cheng B, Titterington DM (1994) Neural Networks: A review from a Statistical Perspective. Statistical Science 9: 2-54.

37. Hosmer D, Lemeshow S (1989) Applied Logistic Regression. J Wiley \& Sons, NY: USA.

38. Miall WE, Oldham PO (1955) A study of arterial blood pressure and its inheritance in a sample of the general population. Clinical Science 14: 459-487.

39. http://sci2s.ugr.es/keel/dataset.php?cod=138\#sub1

40. https://archive.ics.uci.edu/ml/machine-learning-databases/ecoli/ecoli.names

41. Wing W, Yeung DS (2014) Diversified Sensitivity-Based Undersampling for Imbalance Classification Problems. leee Transactions on Cybernetics.

42. Anand A, Pugalenthi G, Fogel GB, Suganthan PN (2010) An approach fo classification of highly imbalanced data using weighting and under-sampling Amino Acids 39:5.

43. Li Q, Wang Y, Bryant S H (2009) A novel method for mining highly imbalanced high-throughput Screening data in PubChem. Bioinformatics 25: 3310-3316.

44. McCarthy K, Zabar B, Weiss GM (2005) Does Cost-Sensitive Learning Beat Sampling for Classifying Rare Classes?. Proc. Int'l Workshop Utility-Based Data Mining, pp: 69-77.

45. Liu XY, Zhou ZH (2006) The influence of class imbalance on cost sensitive learning: An Empirical study. in Proceedings of the 6th ICDM. Washington, DC, USA, pp: 970-974

46. David CA (2009) Finding problems in, proposing solutions to, and performing analysis on imbalanced data. PhD Dissertation, University of Notre Dame, Indiana.

47. Marcellin Simon (2008) Arbres de décision en situation d'asymétrie. Phd Thesis informatique, Université Lumière Lyon II, France.

48. Kotsiantis S, Kanellopoulos D, Pintelas P (2006) Handling imbalanced datasets: A review. GESTS. International Transactions on Computer Science and Engineering 30: 25-36. 